Ann. Génét. Sél. anim., I974, 6 (I), I-I6.

\title{
MISE EN ÉVIDENCE, SUR LES SPERMATOZOÏDES BOVINS, DE QUELQUES ANTIGENES APPARTENANT AU SYSTEME DE GROUPES SANGUINS $S$
}

\author{
C. PRAKASH ( $\left.{ }^{1}\right)$ \\ avec la collaboration technique de Dominique SEITz \\ Laboratoive de Génétique biochimique, \\ Centre national de Recherches zootechniques, I. N. R. A., \\ 78350 Jouy en Josas

\section{RÉSUMÉ}

Nous démontrons dans ce travail que les spermatozoïdes de bovins possédant les facteurs antigéniques érythrocytaires $S, S^{\prime \prime}$ et $U^{\prime}$ du système $S$, absorbent les antisérums correspondants d'une manière beaucoup plus nette que les spermatozoïdes de bovins négatifs pour ces facteurs.

Ce résultat n'a pu être mis en évidence que grâce à l'emploi de notre technique de séparation des spermatozoïdes sur gradient de ficolle, et à la mise au point d'une technique d'hémolyse appropriée.

Le fait que les spermatozoïdes de sujets négatifs pour les facteurs érythrocytaires considérés puissent cependant abaisser l'activité anticorps des antisérums semble imputable à l'activité protéolytique de l'acrosine, sans que le mécanisme exact du phénomène observé ait pu être élucidé.

Les résultats de ce travail sont discutés à la lumière de travaux récents qui semblent avoir établi que l'information génétique portée à l'état haploïde par les spermatozoïdes peut s'exprimer partiellement.

\section{INTRODUCTION}

Le fait que l'information génétique à l'état haploïde dont sont porteurs les spermatozoïdes puisse s'exprimer dans ces cellules, et le fait que l'on puisse envisager d'en déceler l'expression à l'aide de marqueurs antigéniques offre de nouvelles perspectives pour l'amélioration génétique des animaux domestiques.

Des études fondamentales ont été réalisées, dans ce domaine, chez 1'Homme et chez les Rongeurs, où la mise en œuvre de techniques immunologiques élaborées a permis d'admettre que le génome des spermatozoïdes pouvait ne pas être entièrement réprimé. De nombreuses revues générales (METZ, I967 ; BEATTY, I970 ; PAPIvanov et Vulchanov, I97I ; Cohen, I97I ; BeER et Brllingham, r97I ; Shulman, Inde.

(1) Adresse actuelle : Division of Genetics, National Dairy Research Institute, Karnal, Haryana, 
I97 I et I972) ont été consacrées aux travaux antérieurs et à leurs implications possibles en génétique fondamentale, en médecine et dans le contrôle des naissances. Notre interprétation de ces résultats, qui se base sur les données actuelles de l'immunogénétique, a été présentée dans une revue récente (PRAKASH, I974a).

De manière générale, les constituants antigéniques du sperme des mammifères peuvent être classés en quatre catégories principales : les autoantigènes des spermatozoïdes, les alloantigènes des spermatozoïdes, les antigènes du plasma séminal adsorbés sur les spermatozoïdes et les antigènes du plasma séminal.

Les travaux élégants de Vorsin et de ses collaborateurs ont mis en évidence la présence d'autoantigènes sur les spermatozoïdes du cobaye (VOISIN et al., I95I ; VoIsin, I958 ; VoISIn et al., I964 ; VoISIN et Toullet, I967 ; VoISIn et ToulLET, I968). En ce qui concerne les alloantigènes et les antigènes du plasma séminal adsorbés sur les spermatozoïdes, les nombreux travaux publiés jusqu'à présent n'ont pas permis de tirer des conclusions très claires.

Des tentatives de mise en évidence d'antigènes érythrocytaires bovins sur les spermatozoïdes homologues ont été faites à l'aide des méthodes d'absorption par Docton et al. (I952) ; Schmid et al. (I964) ; MATOUSEK (I964). L'interprétation de ces résultats est très délicate par suite de 1'intervention des trois facteurs suivants :

Io présence, dans le culot de centrifugation des spermatozoïdes destinés à l'absorption, de petites quantités de plasma séminal, qui contient un facteur hémolytique important (Millar, I956 ; Hunter et al., I963; Petrovsky, I964 ; MATOUSEK, I970 et I972) ;

$2^{\circ}$ libération d'une forte activité anti-complémentaire (STONE, I972; PRAKASH, I969) au cours de l'absorption ou de la centrifugation ;

$3^{\circ}$ contamination des préparations de spermatozoïdes par des cellules et des débris cellulaires d'origine diploïde, qui peuvent être porteurs de l'antigène en question.

Nous rapportons dans ce travail les premiers résultats de nos recherches visant à mettre en évidence sur les spermatozoïdes bovins certains facteurs antigéniques des systèmes de groupes sanguins. Elles ont porté sur les facteurs du système $\mathrm{S}$ dont le tableau I donne 1'état actuel selon GrosclaudE (I973).

\section{TABLEAU I}

État actuel du système $S$ de groupes sanguins des bovins

(Grosclaude, I973)

Facteurs antigéniques appartenant au système $\mathrm{S}$ :

$\mathrm{S}, \mathrm{U}, \mathrm{H}^{\prime}, \mathrm{U}^{\prime}, \mathrm{H}^{\prime \prime}, \mathrm{U}^{\prime \prime}, \mathrm{S}^{\prime \prime}, \mathrm{U}_{2}^{\prime}{ }^{\left({ }^{1}\right)}$

Phénogroupes du système $\mathrm{S}$ actuellement identifiés :

$$
\begin{aligned}
& \mathrm{H}^{\prime}, \mathrm{SH}^{\prime}, \mathrm{U}^{\prime}, \mathrm{UH}^{\prime} \mathrm{H}^{\prime \prime}, \mathrm{UH}^{\prime} \mathrm{H}^{\prime \prime} \mathrm{U}^{\prime \prime}, \\
& \mathrm{H}^{\prime} \mathrm{U}^{\prime \prime}, \mathrm{H}^{\prime} \mathrm{H}^{\prime \prime}, \mathrm{SH}^{\prime} \mathrm{S}^{\prime \prime}, \mathrm{H}^{\prime} \mathrm{S}^{\prime \prime}, \mathrm{U}^{\prime \prime}, \mathrm{U}_{2}^{\prime}, \mathrm{U}^{\prime \prime} \mathrm{U}_{2}^{\prime}, \\
& \left.\mathrm{UH}^{\prime} \mathrm{U}^{\prime \prime}, \mathrm{H}^{\prime} \mathrm{H}^{\prime \prime} \mathrm{U}^{\prime \prime}, \mathrm{SH}^{\prime} \mathrm{U}^{\prime \prime}, \mathrm{UH}^{\prime},-{ }^{2}\right) .
\end{aligned}
$$

(1) Le facteur $\mathrm{U}_{2}^{\prime}$ n'est pas un sous-groupe linéaire de $\mathrm{U}^{\prime}$; sa dénomination est provisoire.

(2) Phénogroupe "négatif ". 
Nous avons pu mener à bien cette étude après avoir résolu les difficultés mentionnées ci-dessus, qui limitaient les possibilités de la technique d'absorption, et en mettant en œuvre une technique de mesure quantitative de l'activité résiduelle des anticorps, qui nous a permis d’opérer avec une quantité limitée đe spermatozoïdes.

\section{MATÉRIEL E'T MÉTHODES}

$$
\text { I. - Réactifs }
$$

Les réactifs monospécifiques sont ceux couramment utilisés par le service d'analyse des groupes sanguins de l'I. N. R. A. à Jouy en Josas. Ils ont été obtenus par iso-immunisation avec des globules rouges bovins et purifiés par absorption. Ces réactifs ont été soumis à des contrôles de pureté sévères qui ont confirmé leur spécificité.

\section{2. - Origine et collecte du sperme}

Le sperme utilisé provenait de taureaux de race Normande, entretenus au Centre d'Insémination de l'Aigle et dans certains cas, de taureaux de race Normande ou Frisonne, entretenus au C. N. R. Z. Il a été collecté au moyen d'un vagin artificiel et placé dans une bouteille isolante.

\section{3. - Préparation des spermatozoïdes}

Les cellules contaminantes et les débris cellulaires sont éliminés par la technique de gradient de densité sur ficolle (PraKash, I974 b). Les spermatozoïdes sont ensuite lavés trois fois par centrifugation (6oo g-I 5 minutes) dans un grand volume de solution saline physiologique ou de solution saline tamponnée à $\mathrm{pH} 7,2$, à l'aide de phosphate de Sodium et de Potassium. Ils sont comptés à l'aide d'un hémocytomètre et leur concentration est ajustée ; ils sont enfin centrifugés à I 500 g-I 5 minutes. Tous les culots de spermatozoïdes utilisés pour l'absorption ont été contrôlés de façon rigoureuse par l'examen de frottis au microscope à contraste de phase. Seuls ont été retenus, pour l'absorption, ceux qui s'étaient avérés dépourvus de toute contamination par des débris cellulaires. A noter que dans le cas où le sperme provenait de taureaux du Centre de Jouy en Josas, sollicités assez irrégulièrement, la qualité des préparations de spermatozoïdes n'était pas toujours satisfaisante. Cette observation confirme que le prélèvement de la semence à des intervalles irréguliers nuit à une bonne séparation sur gradient de ficolle (PRAKASH, I973 $b$ ).

\section{4. - Technique d'absorption}

La recherche, sur les spermatozoïdes, de chacun des facteurs antigéniques retenus pour cette étude comporte au moins 3 essais :

a) essai d'absorption du réactif correspondant à l'aide des spermatozoïdes d'un taureau dont les érythrocytes possèdent le facteur considéré ;

b) essai d'absorption avec les spermatozoïdes d'un taureau dont les érythrocytes ne possèdent pas ce facteur ;

c) témoin sans spermatozoïdes. Dans certains cas, les essais d'absorption ont été répétés avec les spermatozoïdes de plusieurs taureaux.

Dans tous les cas, les tubes contenant le mélange d'anticorps et de spermatozoïdes sont d'abord incubés à $30^{\circ} \mathrm{C}$ pendant une heure et agités à l'aide d'un agitateur Vortex toutes les I 5 minutes puis placés pendant une nuit (I 2-16 heures) à $+4^{\circ} \mathrm{C}$ sous agitation douce et continue. On effectue ensuite une centrifugation à I 500 g pendant $\mathrm{I} 5$ minutes, à $+4^{\circ} \mathrm{C}$, et les surnageants sont transférés avec précaution, à l'aide d'une pipette Pasteur siliconée, dans d'autres tubes et stockés à $-20^{\circ} \mathrm{C}$. La perte de réactif est, au vu du volume de surnageant récupéré, très faible. Le volume de tous les tubes est cependant réajusté au même niveau, en général o, $3 \mathrm{ml}$. Pour éviter l'évaporation, les tubes sont fermés à l'aide de bouchons recouverts de paraffine.

Après absorption, l'examen du culot de spermatozoïdes par frottis en couche mince, au microscope en contraste de phase, révèle qu'un nombre significatif de spermatozoïdes (entre ro-6o p. Ioo) se trouvent brisés à la jonction de la tête et de la queue. 


\section{5. - Technique d'hémolyse}

La technique d'hémolyse couramment utilisée dans les analyses de groupes sanguins bovins a été suivie au début de notre travail. Nous avons cependant constaté que pour le problème étudié, cette technique était inadéquate, ce qui nous a amené à mettre au point une technique plus élaborée. Cet aspect de notre travail est exposé plus loin, dans la première partie du chapitre "Résultats".

Quelque soit la technique utilisée, des tubes de matière plastique ou de verre siliconé ont été utilisés pour réduire les pertes de cellules et de sérum par adhésion aux parois. Les dilutions de sérum ont été faites à l'aide de la seringue de précision Hamilton, ou de pipettes à cône de matière plastique.

\section{6. - Chromatographie et électrophorèse sur papier}

Les techniques utilisées sont celles décrites par Grosclaude et al. (1970); les détails sont donnés dans les légendes des figures.

\section{RÉSULTATS}

\section{I. - Modification de la technique d'hémolyse}

Au début de notre travail, nous nous sommes contentés de mesurer 1'activité anticorps résiduelle après absorption d'un réactif par un culot de spermatozoïdes en utilisant la technique d'hémolyse couramment pratiquée pour l'analyse des groupes sanguins des bovins. Les résultats les plus représentatifs de ce travail sont présentés dans le tableau 2.

\section{TABLEAU 2}

\section{Absorption de réactifs spécifiques de groupes sanguins bovins par des spermatozoides de taureau}

L'activité anticorps est mesurée par hémolyse selon la technique classique : durée de sensibilisation, $\mathrm{I} / 2 \mathrm{~h}$; lectures visuelles, avec agitation manuelle, $\mathrm{I} / 2 \mathrm{~h}, \mathrm{I} \mathrm{h} \mathrm{I/2}$ et $3 \mathrm{~h}$ après addition du complément de lapin; température, 28 à $30^{\circ} \mathrm{C}$. Le degré d'hémolyse est noté de o (pas d'hémolyse) à 4 (hémolyse totale) selon l'échelle suivante : $0, \operatorname{tr}, \mathrm{I}, 2,3,4$. Le tableau représente, à titre d'exemple, les résultats obtenus avec anti- $\mathrm{S}^{\prime \prime}$.

\begin{tabular}{|c|c|c|c|}
\hline \multirow[b]{2}{*}{$\begin{array}{c}\text { Érythrocytes } \\
\text { utilisés pour le test } \\
\text { d'hémolyse }\end{array}$} & \multicolumn{3}{|c|}{ Degré d'hémolyse noté par trois lectures successives } \\
\hline & $\begin{array}{c}\text { anti-S" } \\
\text { incubé avec les } \\
\text { spermatozoïdes } \\
\text { de taureaux } \\
\mathrm{S}^{\prime \prime} \text { négatifs }\end{array}$ & $\begin{array}{c}\text { anti- } S^{\prime \prime} \\
\text { incubé avec les } \\
\text { spermatozoïdes } \\
\text { de taureaux } \\
S^{\prime \prime} \text { positifs }\end{array}$ & $\begin{array}{c}\text { anti-S" } \\
\text { non incubé } \\
\text { avec des } \\
\text { spermatozoïdes }\end{array}$ \\
\hline $\begin{array}{l}\mathrm{S}^{\prime \prime} \text { positifs } \\
\mathrm{S}^{\prime \prime} \text { négatifs }\end{array}$ & $\begin{array}{l}0,2,4 \\
0, \mathrm{tr}, \mathrm{tr}\end{array}$ & $\begin{array}{l}0,0,0 \\
0, \mathrm{tr}, \mathrm{tr}\end{array}$ & $\begin{array}{l}4,4,4 \\
0, \mathrm{tr}, \mathrm{tr}\end{array}$ \\
\hline
\end{tabular}

Cependant, le protocole de la réaction d'hémolyse classique ne nous a pas semblé représenter les conditions optimales pour notre travail, tant dans le domaine de la sensibilité de la réaction, qu'en ce qui concerne le degré d'hémolyse non spécifique. 
D'autre part, la lecture visuelle directe des réactions d'hémolyse ne permet pas de déceler avec suffisamment de précision les faibles différences auxquelles on peut s'attendre par suite de l'emploi d'un nombre relativement faible de spermatozoïdes. Nous avons enfin vérifié, en ajoutant à nouveau du complément frais 2 à 3 heures après le début de la première addition, que l'on augmentait ainsi de degré d'hémolyse dans les tubes contenant l'anticorps mis en contact avec les spermatozoïdes, alors qu'on n'observait pas ce phénomène dans les tubes témoins. Ce résultat trahit la libération de facteurs anticomplémentaires par les spermatozoïdes durant l'absorption. Nous avons donc été amenés à apporter un certain nombre de perfectionnements à notre protocole.

\section{TABLEAU 3}

Effets de la durée de sensibilisation, de la durée de l'hémolyse et de la température pendant ces deux phases sur le degré d'hémolyse d'érythrocytes bovins par les immunsérums spécifiques correspondants (anti- $\mathrm{U}^{\prime}$ et anti- $\mathrm{H}^{\prime \prime}$ ).

Le degré d'hémolyse est exprimé, en pourcentage, par un index d'hémolyse (voir texte). A l'exception du lavage après sensibilisation qui n'a pas été effectué ici, le protocole expérimental est celui qui est décrit dans la figure r. Les séries (chiffres latins) correspondent à des essais faits un même jour.

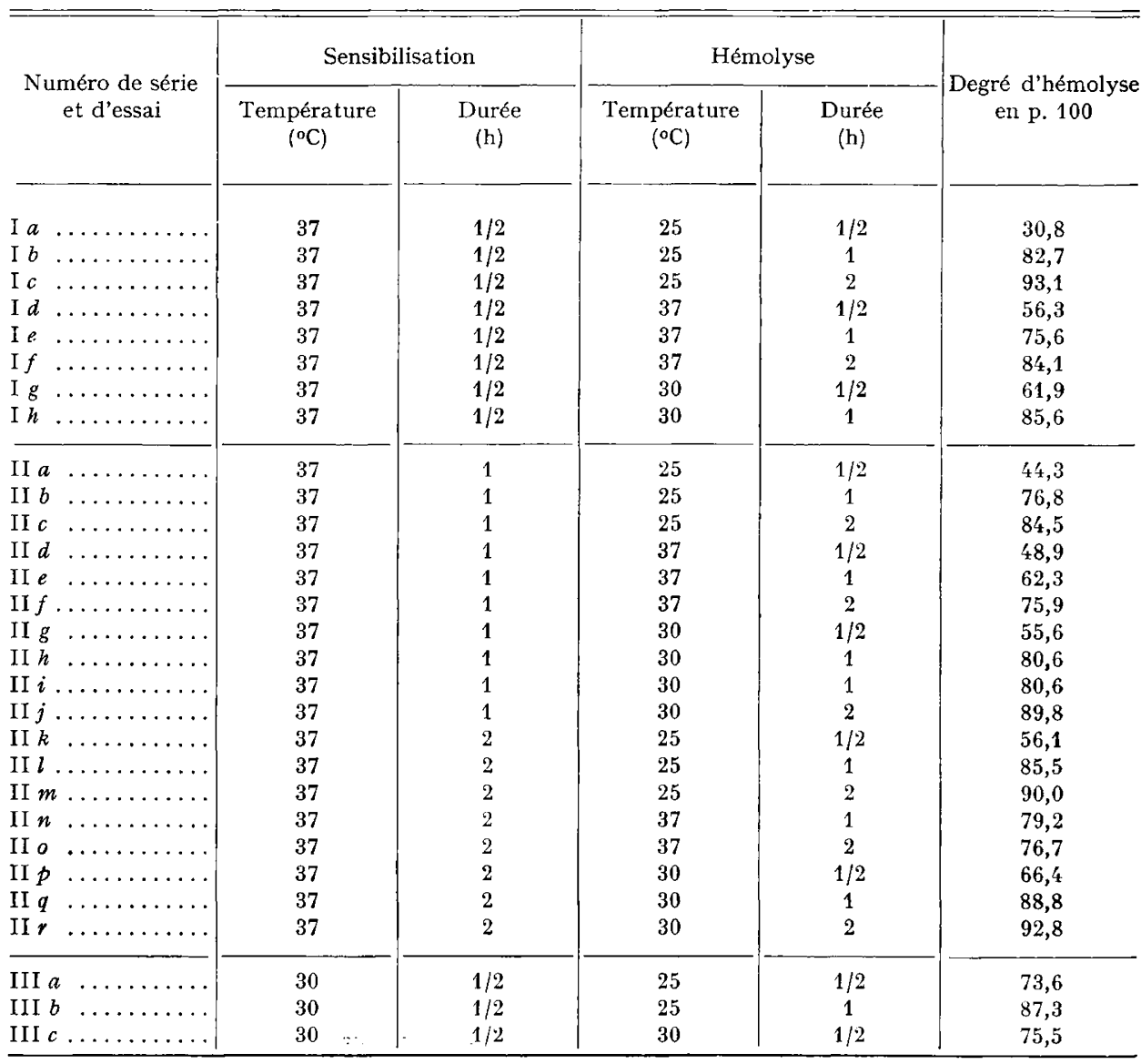


TABLEAU 3 (suite)

\begin{tabular}{|c|c|c|c|c|c|}
\hline \multirow{2}{*}{$\begin{array}{c}\text { Numéro de série } \\
\text { et d'essai }\end{array}$} & \multicolumn{2}{|c|}{ Sensibilisation } & \multicolumn{2}{|c|}{ Hémolyse } & \multirow{2}{*}{$\begin{array}{l}\text { Degré d'hémolyse } \\
\text { en p. } 100\end{array}$} \\
\hline & $\begin{array}{l}\text { Température } \\
\left({ }^{\circ} \mathrm{C}\right)\end{array}$ & $\begin{array}{c}\text { Durée } \\
\text { (h) }\end{array}$ & $\begin{array}{l}\text { Température } \\
\left({ }^{\circ} \mathrm{C}\right)\end{array}$ & $\begin{array}{l}\text { Durée } \\
\text { (h) }\end{array}$ & \\
\hline $\begin{array}{l}\text { III } d \quad \ldots \ldots \ldots \\
\text { III } e \ldots \ldots \ldots \\
\text { III } f \ldots \ldots \\
\text { III } g \\
\text { III } h \\
h\end{array}$ & $\begin{array}{l}30 \\
25 \\
25 \\
25 \\
25\end{array}$ & $\begin{array}{l}1 / 2 \\
1 / 2 \\
1 / 2 \\
1 / 2 \\
1 / 2\end{array}$ & $\begin{array}{l}30 \\
25 \\
25 \\
30 \\
30\end{array}$ & $\begin{array}{c}1 \\
1 / 2 \\
1 \\
1 / 2 \\
1\end{array}$ & $\begin{array}{l}82,7 \\
71,9 \\
94,8 \\
76,7 \\
97,0\end{array}$ \\
\hline 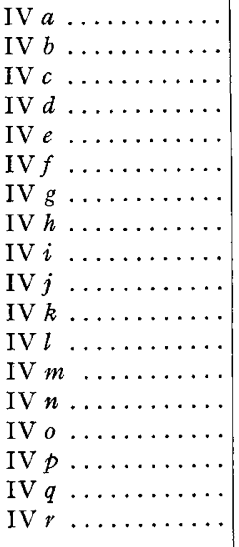 & $\begin{array}{l}30 \\
30 \\
30 \\
30 \\
30 \\
30 \\
30 \\
30 \\
30 \\
30 \\
30 \\
30 \\
30 \\
30 \\
30 \\
30 \\
30 \\
30\end{array}$ & $\begin{array}{c}1 \\
2 \\
1 \\
2 \\
1 / 2 \\
1 \\
2 \\
1 \\
2 \\
1 \\
2 \\
1 / 2 \\
1 \\
2 \\
1 / 2 \\
1 \\
2 \\
1\end{array}$ & $\begin{array}{l}25 \\
25 \\
25 \\
25 \\
25 \\
25 \\
25 \\
30 \\
30 \\
30 \\
30 \\
30 \\
30 \\
30 \\
37 \\
37 \\
37 \\
37\end{array}$ & $\begin{array}{c}1 / 2 \\
1 / 2 \\
1 \\
1 \\
2 \\
2 \\
2 \\
1 / 2 \\
1 / 2 \\
1 \\
1 \\
2 \\
2 \\
2 \\
1 / 2 \\
1 / 2 \\
1 / 2 \\
1\end{array}$ & $\begin{array}{l}86,5 \\
88,5 \\
90,2 \\
93,9 \\
95,0 \\
92,2 \\
96,6 \\
87,2 \\
89,9 \\
90,2 \\
96,8 \\
91,8 \\
91,2 \\
94,2 \\
78,0 \\
84,8 \\
90,2 \\
88,6\end{array}$ \\
\hline 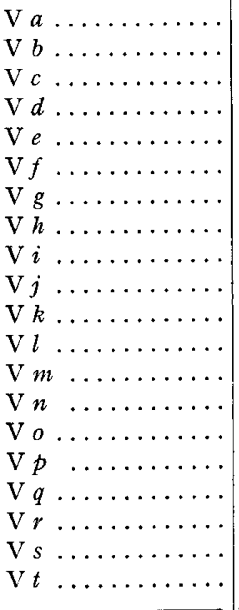 & $\begin{array}{l}25 \\
25 \\
25 \\
25 \\
25 \\
25 \\
25 \\
25 \\
25 \\
25 \\
25 \\
25 \\
25 \\
25 \\
25 \\
25 \\
25 \\
25 \\
25 \\
25\end{array}$ & $\begin{array}{c}1 \\
2 \\
1 \\
2 \\
1 / 2 \\
1 \\
2 \\
1 \\
2 \\
1 \\
2 \\
1 / 2 \\
1 \\
2 \\
1 / 2 \\
1 \\
2 \\
1 / 2 \\
1 \\
2\end{array}$ & $\begin{array}{l}25 \\
25 \\
25 \\
25 \\
25 \\
25 \\
25 \\
30 \\
30 \\
30 \\
30 \\
30 \\
30 \\
30 \\
37 \\
37 \\
37 \\
37 \\
37 \\
37\end{array}$ & $\begin{array}{c}1 / 2 \\
1 / 2 \\
1 \\
1 \\
2 \\
2 \\
2 \\
1 / 2 \\
1 / 2 \\
1 \\
1 \\
2 \\
2 \\
2 \\
1 / 2 \\
1 / 2 \\
1 / 2 \\
1 \\
1 \\
1\end{array}$ & $\begin{array}{l}27,2 \\
26,2 \\
66,1 \\
72,9 \\
80,2 \\
80,3 \\
86,2 \\
72,6 \\
80,9 \\
91,4 \\
94,2 \\
94,3 \\
92,0 \\
94,7 \\
53,3 \\
70,8 \\
70,6 \\
75,2 \\
85,5 \\
87,7\end{array}$ \\
\hline 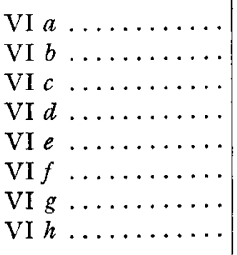 & $\begin{array}{l}25 \\
30 \\
37 \\
25 \\
25 \\
25 \\
30 \\
25\end{array}$ & $\begin{array}{c}1 / 2 \\
2 \\
1 / 2 \\
1 / 2 \\
1 \\
2 \\
2 \\
2\end{array}$ & $\begin{array}{l}25 \\
25 \\
25 \\
30 \\
30 \\
30 \\
30 \\
30\end{array}$ & $\begin{array}{l}1 \\
2 \\
2 \\
1 \\
1 \\
1 \\
1 \\
2\end{array}$ & $\begin{array}{l}55,1 \\
84,7 \\
89,2 \\
58,7 \\
95,6 \\
84,4 \\
81,4 \\
83,8\end{array}$ \\
\hline
\end{tabular}


Nous avons tout d'abord étudié systématiquement les effets, sur la réaction d'hémolyse, de la durée de sensibilisation des érythrocytes, de la durée de la phase d'hémolyse proprement dite, et de la température. Nos résultats, résumés dans le tableau 3, ainsi que certaines considérations d'ordre pratique (souci de ne pas allonger inutilement la durée de réaction, ni de multiplier les agitations) nous ont conduit à retenir comme optimales, les conditions suivantes : sensibilisation : I heure à $25^{\circ} \mathrm{C}$; hémolyse : I heure à $30^{\circ} \mathrm{C}$. D'autre part, pour éliminer autant que possible l'activité anticomplémentaire provenant des spermatozoïdes brisés au cours de l'absorption de l'antisérum, les érythrocytes sont lavés après la phase de sensibilisation. Ennfin, la lecture des résultats de la réaction d'hémolyse est faite avec précision, au spectrophotomètre (tabl. 4).

\section{TABLEAU 4}

Technique d'hémolyse immune mise au point pour ce travail

- Mélange de 0,05 ml d'une suspension d'hématies à 2,5 p. 100 ajustée par spectrophotométrie et de 0,10 ml d'immunsérum dilué dans $\mathrm{NaCl} 0,156 \mathrm{M}$ contenant 5 p. 100 de sérum albumine bovine (SAB). Incubation à $25^{\circ} \mathrm{C}$ pendant 1 heure, avec agitation tous les quarts d'heure.

- Addition de $5 \mathrm{ml}$ de $\mathrm{NaCl} 0,156 \mathrm{M}$ contenant 0,1 p. 100 de $\mathrm{SAB}$ et centrifugation à $600 \mathrm{~g}$ pendant $10 \mathrm{mn}$ puis à $2000 \mathrm{~g}$ pendant $5 \mathrm{mn}$; élimination du surnageant.

- Addition à $0^{\circ} \mathrm{C}$ de $0,05 \mathrm{ml}$ de complément, constitué de 9 parties de sérum de lapin pour 1 partie de sérum de cobaye.

- Incubation à $30^{\circ} \mathrm{C}$ pendant 1 heure, avec agitation tous les quarts d'heure.

- Addition de $4 \mathrm{ml}$ de $\mathrm{NaCl} 0,156 \mathrm{M}$ contenant 0,1 p. 100 de SAB, mélange, puis centrifugation dans les mêmes conditions que plus haut.

- Mesure de l'absorption donnée par le surnageant à $413 \mathrm{~nm}$.

Ce protocole comporte deux témoins différents :

a) témoin sans érythrocytes ni antisérum $(0,05 \mathrm{ml}$ de complément et $4 \mathrm{ml}$ de tampon) pour réglage du spectrophotometre.

b) témoin où l'antisérum est remplacé par du tampon pour correction du degré d'hémolyse.

En ce qui concerne la présentation des résultats, 1'utilisation d'un index hémolytique analogue à l'index de spermatotoxicité de HAMmERLYNCK et RUMKE (I968) permet d'éliminer les effets de l'hémolyse non spécifique.

\section{2. - Absorption des réactifs de groupes sanguins par des spermatozoïdes}

Les résultats de nos essais d'absorption de réactifs de groupes sanguins bovins par des spermatozoïdes de la même espèce, non contaminés par du plasma séminal ni par des cellules ou débris cellulaires d'origine diploïde, sont représentés dans les figures I à 3 .

Ces résultats nous semblent prouver que les spermatozoïdes de bovins possédant certains facteurs antigéniques érythrocytaires du système $\mathrm{S}$ sont capables d'absorber les immunsérums spécifiques de ces facteurs. Toutefois, on constate que les spermatozoïdes de sujets ne possédant pas ces facteurs antigéniques provoquent égale- 


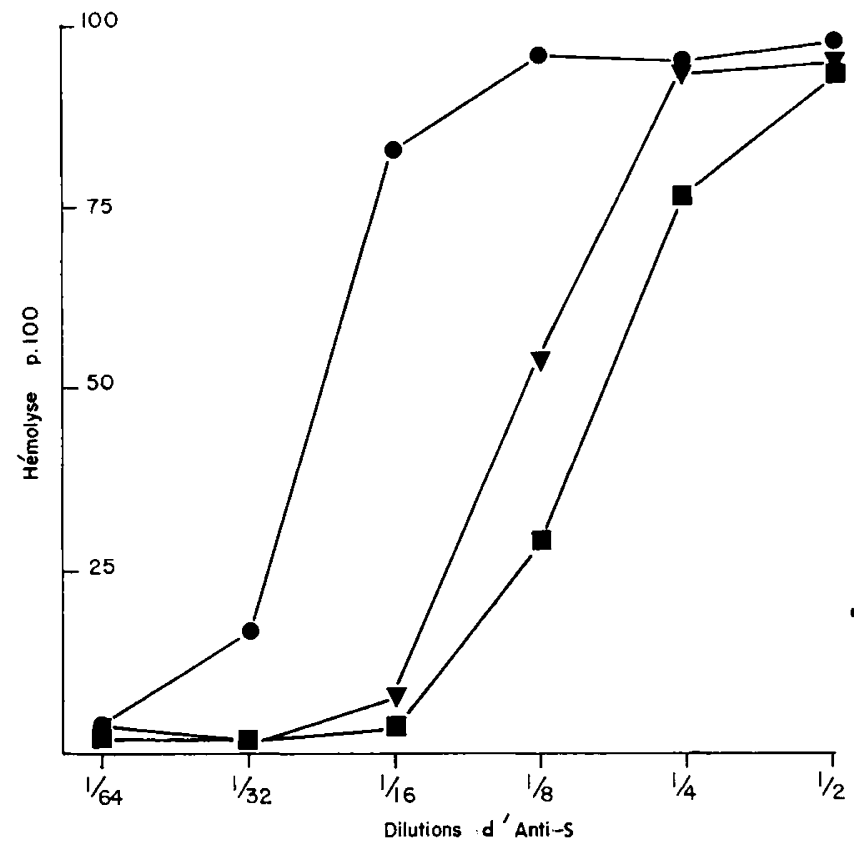

FIG. I. - Absorption du réactif anti-S par des spermatozoïdes

- : réactif non absorbé ;

- : réactif absorbé par les spermatozoïdes de donneurs possédant le facteur érythrocytaire $\mathrm{S}$;

$\nabla$ : réactif absorbé par les spermatozoīdes de donneurs ne possédant pas le facteur $\mathrm{S}$.

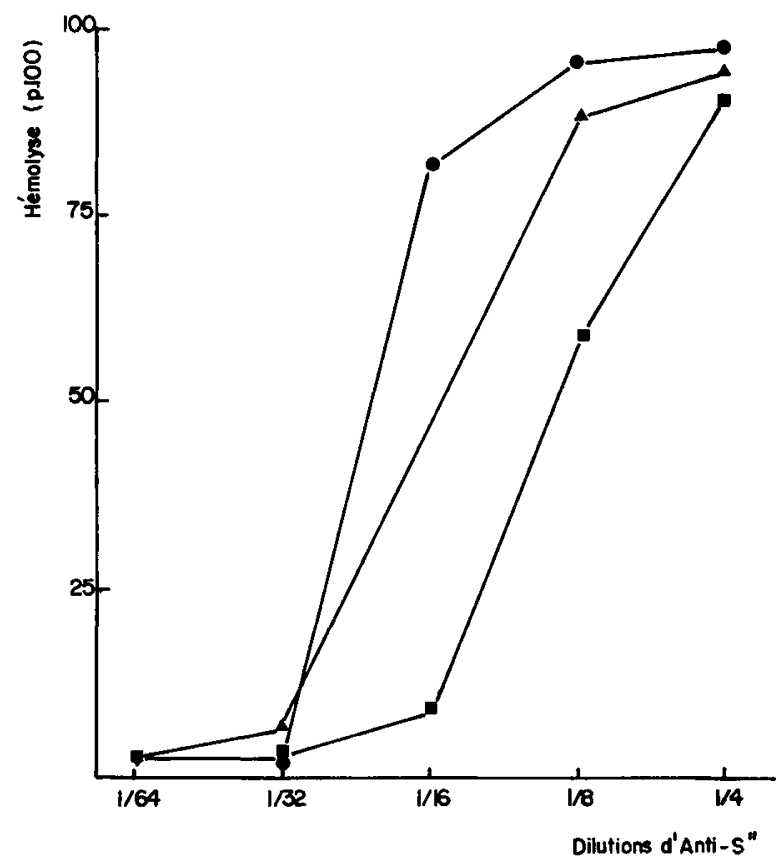

FIG. 2. - Absorption du réactif anti-S" par des spermatozoïdes

- : réactif non absorbé;

- : réactif absorbé par les spermatozoïdes de donneurs possédant le facteur érythrocytaire $\mathrm{S}^{\prime \prime}$.

$\nabla$ : réactif absorbé par les spermatozoïdes de donneurs ne possédant pas le facteur $\mathrm{S}^{\prime \prime}$. 
ment une certaine réduction de l'activité anticorps de l'immunsérum. Sachant qu'à l'inverse des érythrocytes, les spermatozoïdes peuvent dégrader assez fortement les anticorps, nous avons fait l'hypothèse d'une protéolyse, par les spermatozoïdes, des anticorps non spécifiques, hypothèse que nous avons cherché à vérifier.

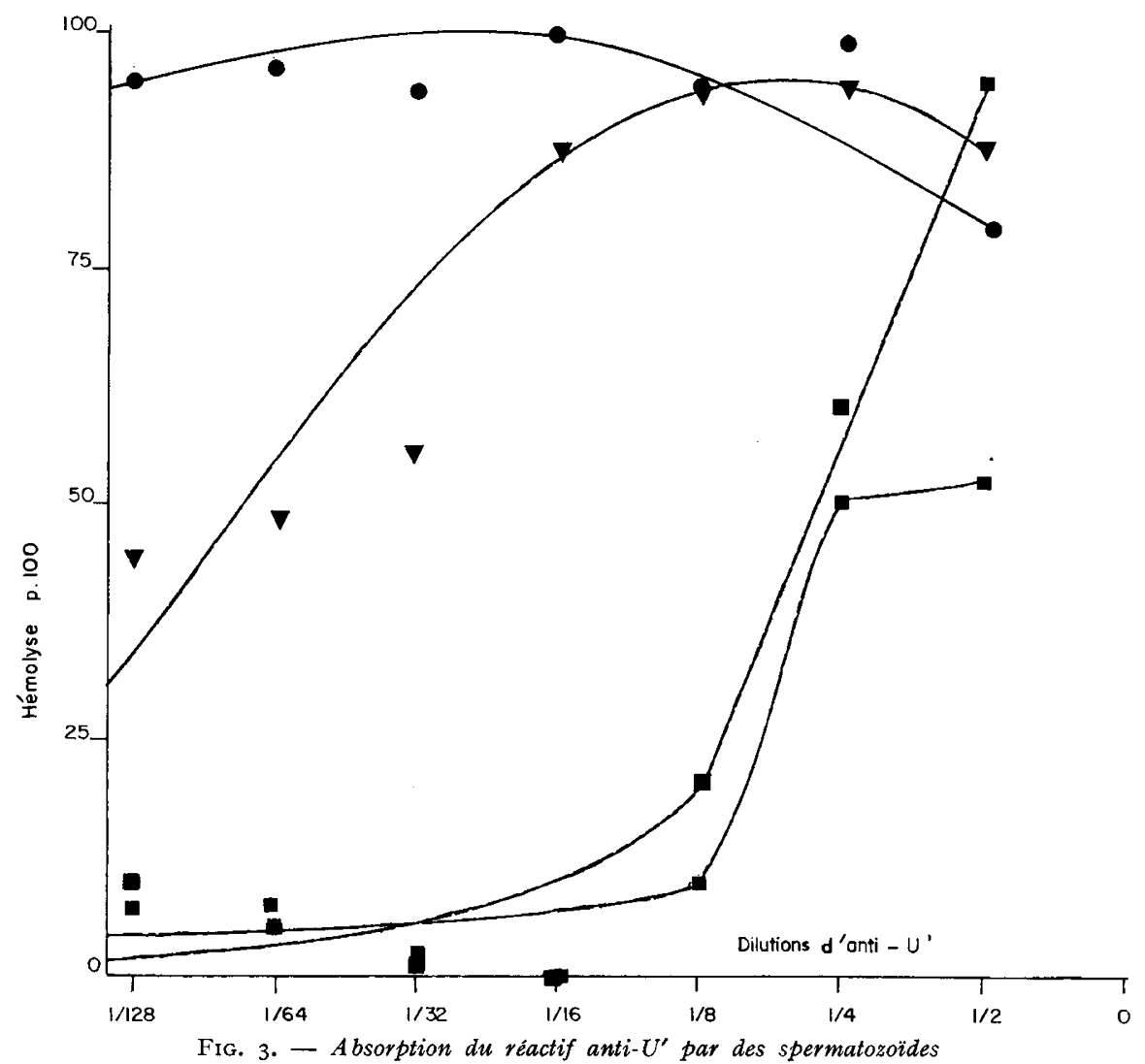

- : réactif non absorbé ;

- : réactif absorbé par les spermatozoïdes de donneurs possédant le facteur érythrocytaire $\mathrm{U}^{\prime}$;

: réactif absorbé par les spermatozoïdes de donneurs ne possédant pas le facteur 'U'.

Notons, auparavant, que l'antisérum absorbé par les spermatozoïdes n'a pas provoqué d'hémolyse non spécifique des érythrocytes bovins, ce qui prouve que grâce à l'efficacité de notre technique de séparation des spermatozoïdes, ces derniers ne sont pas contaminés par du plasma séminal.

\section{3. - Étude de la protéolyse, par les spermatozoïdes, des anticorps non spécifiques}

Nous avons retenu comme substrat, pour cette étude, la caséine bovine qui, selon Multamaki et NiEmI (I972) est un bon substrat. La figure 4 montre qu'en présence de caséine $\alpha_{\delta_{1}}$ bovine, et dans les mêmes conditions que celles de 1'absorption des réactifs de groupes sanguins, l'équipement enzymatique des spermatozoïdes fait preuve d'une forte activité protéolytique. La carte peptidique obtenue dans ces 


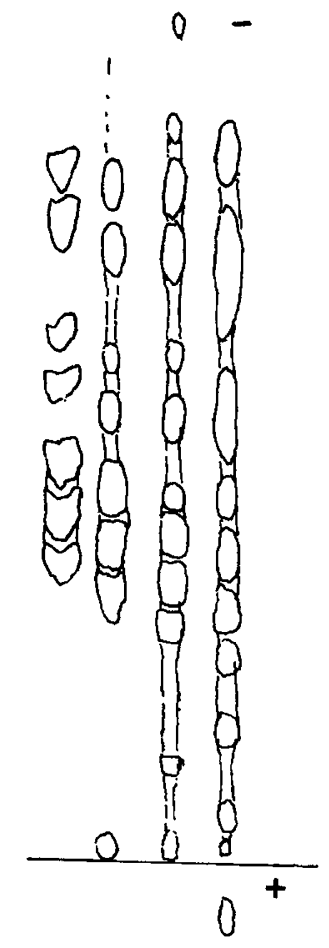

\section{$\begin{array}{llll}1 & 2 & 3 & 4\end{array}$}

Fig. 4. - Protéolyse due aux spermatozoüdes dans les conditions d'absorption utilisées dans ce travail I : Mélange standard d'acides aminés ; 2 : Résultat de l'incubation des spermatozoïdes dans le tampon; 3: Résultat de l'incubation de la caséine avec les spermatozoïdes; 4 : Hydrolysat trypsique de la caséine $\alpha_{s_{1}}$ bovine $\left(\mathrm{E} / \mathrm{S}: \mathrm{I} / 200 ; 37^{\circ} \mathrm{C} ; 4 \mathrm{~h}\right.$ ). Électrophorèse en tampon formique-acétique, pH I, 9 à 3000 volts pendant $30 \mathrm{mn}$. Coloration à la ninhydrine. Dans les essais 3 et 4 , la caséine non hydrolysée est précipitée par acidification à $\mathrm{pH}_{4}, 6$ et centrifugation à $2000 \mathrm{~g}$ pendant $\mathrm{x}_{5} \mathrm{mn}$.
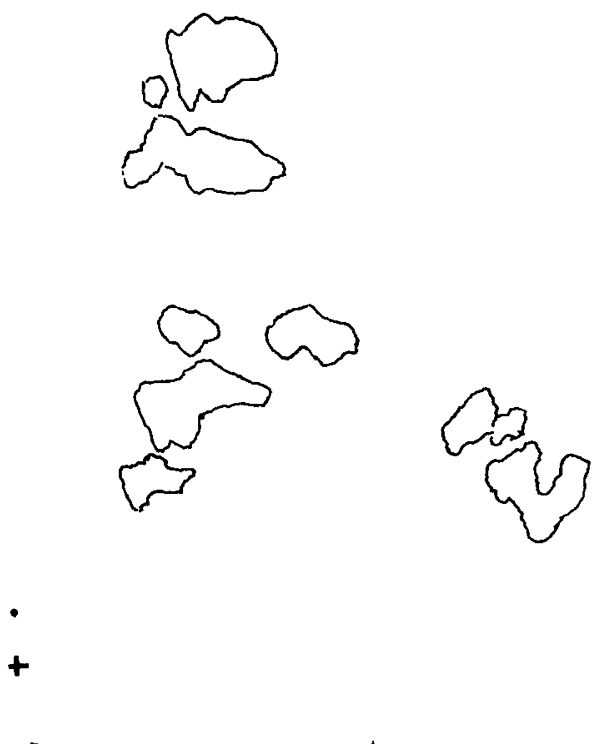

FIG. 5. - Fingerprint des peptides libérés au cours d'une absorption par des spermatozoïdes Mêmes conditions d'incubation et d'électrophorèse que dans le cas de la figure 4. Chromatographie pendant I $2 \mathrm{~h}$ en tampon butanol-pyridine-acide acétique-eau ( $120: 80: 24: 96)$. Coloration à la ninhydrine. 
C

000

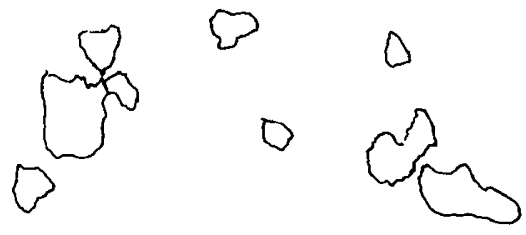

0

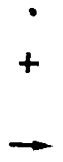

FIG. 6. - Fingerprint des peptides libérés

au cours de l'hydrolyse de la caséine $\alpha_{\delta_{1}}$ bovine par des spermatozoïdes
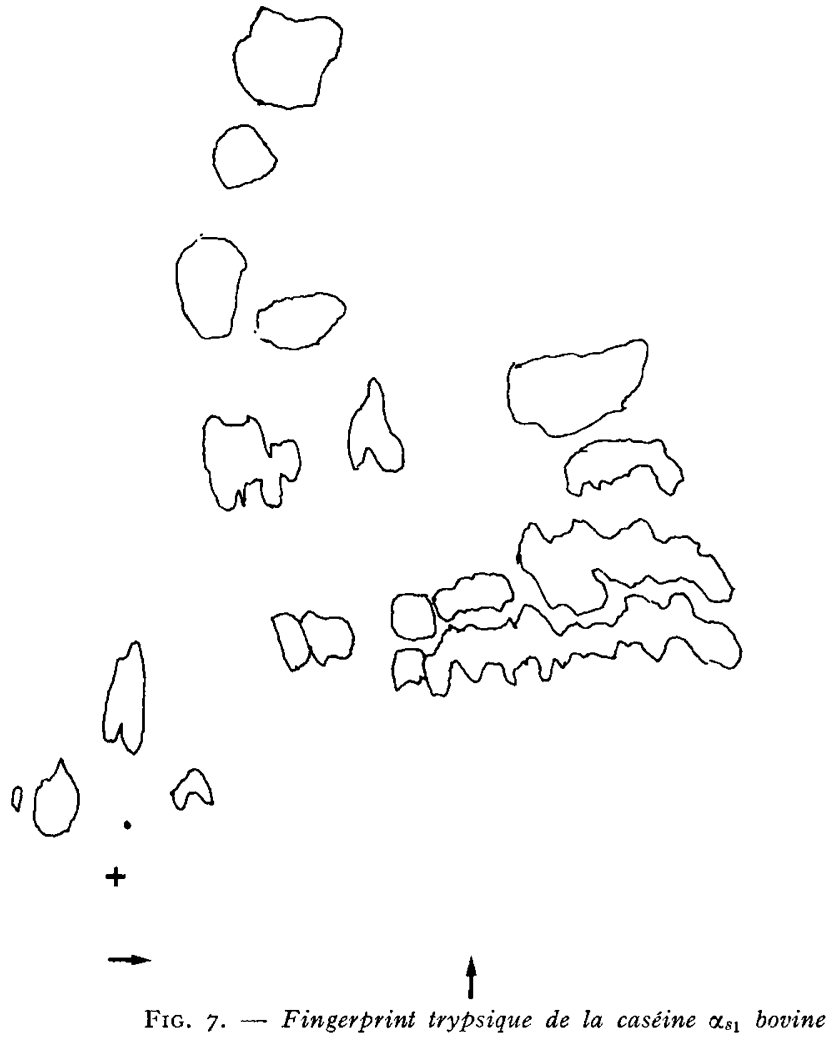
conditions est nettement différente de celle résultant de 1'hydrolyse trypsique de la même caséine (fig. 5, 6 et 7). Le chauffage des spermatozoïdes dans un bain à $100^{\circ} \mathrm{C}$ pendant Io minutes détruit en grande partie cette activité protéolytique.

On sait que la trypsine hautement purifiée coupe la molécule d'immunoglobuline en deux fragments, dits $\left(\mathrm{Fab}^{\prime}\right) 2$ et $\mathrm{F}^{\prime}$. Les fragments $\left(\mathrm{Fab}^{\prime}\right) 2$ peuvent bloquer les sites antigéniques des érythrocytes sans que le complément puisse ensuite intervenir, par suite de la perte du fragment $\mathrm{Fc}$, ce qui empêche finalement l'hémolyse. Nous avons essayé d'hémolyser avec un antisérum (anti- $\mathrm{S}^{\prime \prime}$ ) les érythrocytes restés intacts après une tentative d'hémolyse à l'aide du même sérum préalablement incubé en présence de spermatozoïdes d'un donneur négatif. En utilisant les témoins appropriés, nous avons été amenés à conclure que la protéolyse des anticorps par les spermatozoïdes s'opère différemment de celle qui est obtenue avec la trypsine (tabl. 5).

\section{TABLEAU 5}

Démonstration que les sites antigéniques des érythrocytes restent accessıbles aux anticorps spécifiques correspondants après que ces érythrocytes aient été mis en contact avec un antisérum préalablement incubé avec des spermatozoïdes.

L'antisérum utilisé est anti-S", dilué $1 / 16^{\circ}$

Le degré d'hémolyse est exprimé en p. Ioo (voir texte)

\begin{tabular}{|c|c|c|c|}
\hline & \multicolumn{3}{|c|}{ Degré d'hémolyse } \\
\hline & $\begin{array}{c}\text { anti-S" } \\
\text { incubé avec des } \\
\text { spermatozoïdes } \\
\text { de sujets } \\
\mathrm{S}^{\prime \prime} \text { positifs }\end{array}$ & $\begin{array}{c}\text { anti-S" } \\
\text { incubé avec des } \\
\text { spermatozoïdes } \\
\text { de sujets } \\
\mathrm{S}^{\prime \prime} \text { négatifs }\end{array}$ & $\begin{array}{c}\text { anti-S" } \\
\text { non incubé } \\
\text { avec des } \\
\text { spermatozoïdes }\end{array}$ \\
\hline $\begin{array}{l}\text { I. Hémolyse rési- } \\
\text { duelle (corrigée) }\end{array}$ & 60,0 & 89,0 & 96,1 \\
\hline $\begin{array}{l}\text { II. Hémolyse, par } \\
\text { addition d'anti- } \\
\mathrm{S}^{\prime \prime} \text {, des érythro- } \\
\text { cytes restés in- } \\
\text { tacts dans I }\end{array}$ & 38,0 & 8,0 & 3,0 \\
\hline $\begin{array}{l}\text { Hémolyse totale } \\
\text { en p. } 100\end{array}$ & 98,0 & 97,0 & 99,1 \\
\hline
\end{tabular}

\section{DISCUSSION}

A l'aide de techniques que nous avons mises au point pour cette étude, nous avons montré la présence des facteurs antigéniques $S, S^{\prime \prime}$ et $U^{\prime}$, appartenant au système de groupes sanguins $\mathrm{S}$ bovins, sur les spermatozoïdes de taureaux dont les érythrocytes portent ces facteurs. Nos données indiquent cependant que la densité des sites antigéniques sur les spermatozoïdes doit être plus faible que sur les érythrocytes. Par 
ailleurs, le fait que les spermatozoïdes de sujets ne possédant pas un facteur antigénique donné semblent néanmoins capables d'abaisser en partie l'activité anticorps du réactif correspondant, pose encore un problème majeur ; nous avons supposé que ce phénomène était dû à une hydrolyse partielle des anticorps par l'équipement enzymatique des spermatozoïdes.

ZANEVELD et al. (I973) ont fourni des informations intéressantes sur l'enzyme protéolytique de 1'acrosome des spermatozoïdes, qu'ils ont proposé de nommer " acrosine " au lieu de "trypsin-like enzyme ". Nos résultats, qui montrent une nette différence de spécificité entre la trypsine et l'acrosine vont dans le même sens que ceux de ces auteurs. On notera également que nous n'avons pu éliminer complètement, à l'aide d'un inhibiteur de la trypsine, l'abaissement de 1'activité anticorps provoquée par les spermatozoïdes de sujets ne possédant pas le facteur antigénique correspondant.

Les résultats de ce travail vont à l'encontre de ceux d'autres auteurs (MATouSEK, I970 ; SCHMID et al., I956) qui ont conclu à 1'absence des facteurs antigéniques du système $S$ sur les spermatozoïdes; en fait, les techniques utilisées par ces auteurs n'étaient pas assez sensibles pour déceler, sur les spermatozoïdes, une densité de sites antigéniques relativement faible.

Le problème fondamental que de nombreuses études - y compris la nôtre ont essayé de résoudre, en cherchant à retrouver sur les spermatozoïdes des antigènes détectés sur d'autres cellules est de savoir si une partie, si minime soit-elle, du génome du spermatozoïde, est fonctionnelle. En fait, il semble actuellement établi que sont présents, à la surface des spermatozoïdes humains, des antigènes du système d'histocompatibilité HL-A (FELLOUs et DAUSSET, I970) et à la surface des spermatozoïdes de souris, des antigènes d'histocompatibilité H-2 (VojTISKova et al., I969 et GolDBERG et al., I970), " l'antigène mâle " $\mathrm{Y}$ (GoldBBER et al., I97I) et les produits antigéniques du locus T (GOLDBERG, I972).

Dans le Laboratoire de VoIsIn (I973), des recherches visant à déceler, par la méthode d'immunofluorescence, des autoantigènes au cours de la spermatogenèse du cobaye ont montré que ces autoantigènes n'apparaissent qu'au stade du spermatocyte II. Ce dernier correspond à la dernière étape de la méiose. BEATTY (I972) a émis l'hypothèse d'une mise en activité de gènes du spermatozoïde — gènes qui sont donc à l'état haploïde - en se basant sur les travaux de microscopie électronique de FAWCE'TT (I972). EDELMAN et MILLETTE (I97I) ont montré, à l'aide de la concavaline $\mathrm{A}$, l'existence, sur les spermatozoïdes de souris, de récepteurs glucidiques appartenant vraisemblablement à des glycoprotéines, le nombre de sites récepteurs étant voisin de celui des cellules somatiques. En raison de la distribution asymétrique de ces sites EDELMAN et MILLETTE suggèrent qu'ils représentent bien des éléments de la surface cellulaire.

Les travaux récents de Premkuma et Bhargava (I972) ont montré qu'on n'observe de transcription et de traduction chez les spermatozoïdes bovins qu'à partir de l'ADN haploïde des mitochondries, uniquement présentes dans la pièce intermédiaire; l'ADN nucléaire reste inerte.

Le fait que certains auteurs aient conclu à l'absence d'antigènes de groupes sanguins (par exemple) sur les spermatozoïdes, pourrait s'expliquer par l'intervention du plasma séminal : ce dernier contient, en effet, un inhibiteur de la synthèse de 1'ARN (BHARGAVA, I97I) et la présence de la ribonucléase active y a été signalée (LIBONATI 
et FLORIDI, I969). SHEID et WILSON (I970 $a$, I970 $b$, I97 I $a$, I97I $b$ ) ont mis en évidence une forte inhibition de la méthylation de l'ARN de transfert par la dégradation en polynucléotides du substrat de ces ARN. Ces mêmes auteurs ont également fait la preuve d'une hydrolyse de tous les ARN en présence de plasma séminal. L'ADN mitochondrial qui, dans les spermatozoïdes, porte une copie d'une partie du génome nucléaire, pourrait-il s'exprimer dans certaines conditions? Le fait que les récepteurs des lymphocytes de type $\mathrm{B}$ soient renouvelés toutes les 4 à 5 heures (Loor $e t$ al., I972) montre l'importance qu'ont les propriétés des membranes dans la défense de ces cellules vis-à-vis des lymphocytes immunocompétents de type $T$ du même organisme. L'absence de marqueurs d'histocompatibilité sur les spermatozoïdes pourrait amener leur destruction rapide dans le tractus génital mâle avant l'éjaculation. Il serait donc intéressant de rechercher si 1'ADN mitochondrial ne comporte pas une copie des cistrons commandant les antigènes d'histocompatibilité.

Certains de nos résultats non publiés suggèrent la possibilité que les antigènes du système $S$ de groupes sanguins bovins soient également présents à la surface des lymphocytes. Le système $\mathrm{S}$ pourrait donc être un des systèmes mineurs d'histocompatibilité de l'espèce bovine, mais des études complémentaires sont nécessaires avant de pouvoir l'affirmer.

Rę̧u pour publication en décembre 1973.

\section{REMERCIEMENTS}

Je remercie M. F. Grosclaude, Directeur du Laboratoive de Génétique Biochimique, pour l'aide qu'il m'a apportée dans les domaines scientifique et matériel, le Dr C. R. BALAKRISHNAN, National Dairy Institute, Karnal, Haryana, Inde, pour les discussions que nous avons pu avoir sur les problèmes techniques soulevés par cette étude, ainsi que MM. R. CAssou et R. TASSERY, du Centre d'Insémination artificielle de l'Aigle, pour leurs dons de semence bovine. Je suis également reconnaissant à M. G. Houlier et Mme Marie-Françoise MAHÉ du Laboratoire de Génétique Biochimique de l'aide qu'ils m'ont apportée au cours de ce travail, ainsi qu'à M. H. T. Duc, Laboratoive d'Immunologie de la Transplantation, Hôpital Saint-Antoine, Paris, d'avoir assuré la traduction du manuscrit anglais.

\section{SUMMARY}

\section{DEMONSTRATION OF SOME OF THE AN'TIGENS OF THE S BLOOD GROUP SYSTEM IN BOVINE SPERMATOZOA}

Consistent quantitative differences were found in the capacity of spermatozoa from positive and negative donors for $\mathrm{S}, \mathrm{S}^{\prime \prime}$ and $\mathrm{U}^{\prime}$ antigens of the bovine $\mathrm{S}$ system to absorb rigorously controlled monospecific antisera corresponding to these antigens.

New techniques or modifications of the earlier known techniques were employed. To obviate the interference from non-sperm cells and cellular debris found in ejaculated semen, a density gradient technique on ficoll was used to remove these contaminations from spermatozoa intended for immunological tests. A quantitative hemolytic technique with precise measurement of hemolysis by spectrophotometric methods of residual antibodies after absorption with spermatozoa was used along with the classical techniques.

It was not possible to remove completely the hindrance by proteolytic activity of the acrosin on the antibodies employed in the immunological tests. However, appropriate controls and precise volumetrical measurements have permitted meaningful interpretation of the results.

These results were discussed in the light of the recent relevant experiments wich lend support to the possibility of the partial expression of haploid genome of the spermatozoa. 


\section{RÉFÉRENCES BIBLIOGRAPHIQUES}

Beatty R. A., I970. The genetics of the mammalian gamete. Biol. Rev., 45, 73-Ir9.

BeAtTy R. A., 1972. Dans la discussion de FAwcetT D. W., 1972. Observations on cell differentiation and organelle continuity in spermatogenesis in Proc. Int. Symp. The Genetics of the spermatozoon. Beatty R. A., and Gluecksohn-Waelsch S. ed., Univ. Edinburgh. 37-68.

Beer A. E., Billingham R. E., I97 I a. Immunobiology of mammalian reproduction, in Dixon F. J. and Kunkel H. G., ed. Adv. Immunol., 14, I-84.

Bhargava P. M., r97I. Communication personnelle.

Cohen J., I971. The comparative physiology of gamete population. Adv. Comp. Physiol. and Biochem., 4, 268-380.

Docton F. L., Ferguson L. C., Lazear E. J., Ely F., r952. The antigenicity of bovine spermatozoa. J. Dairy Sci., 35, 706-709.

Edelman G. M., Millette C. F., 197i. Molecular probes of spermatozoan structures. Proc. Natl. Acad. Sci., 68, 2436-2440.

FAwcetr D. W., I972. Observations on cell differentiation and organelle continuity in spermatogenesis. in Proc. Int. Symp. The Genetics of the Spermatozoon. Beatty R. A., and Gluecksohn-Waelsch S. ed., Univ. Edinburgh, 37-68.

Fellous M., Dausset J., r97o. Probable haploid expression of HL-A antigens on human spermatozoon. Nature, London, 225, r9x-r93.

Grosclaude F., Mercier J.-C., Ribadeau-Dumas B., i97o. Structure primaire de la caséine $\alpha_{s_{\mathbf{Y}}}$ bovine. Localisation des peptides trypsiques dans les fragments obtenus par hydrolyse trypsique de la caséine maleylée. Eur. J. Biochem., 14, 98-107.

Grosclaude F., I973. Communication personnelle.

Goldberg E. H., Aoki T., Boyse E. A., Bennett D., i97o. Detection of H-2 antigens on mouse spermatozoa by the cytotoxicity test. Nature, 228, 570-572.

Goldberg E. H., Boyse E. A., Bennett D., Scheid M., Carswell E. A., I97i. Serological demonstration of $\mathrm{H}-\mathrm{Y}$ (male) antigens on mouse sperm. Nature, 232, 478-480.

Goldberg E. H., I972. Ph. D. thesis. Cornell University Dissertation. Abs. Int. Series B, 32 Part I, 6850 B-685I B.

GULLBRING B., 1957. Investigation on the occurrence of blood group antigens from man and serological demonstration of the segregation of characters. Acta. Medica. Scand., 159, 169-I72.

HAMERLynck J., RUmke P., I968. A test for the detection of cytotoxic antibodies to spermatozoa in man. J. Reprod. Fert., 17, I9I-I94.

Hunter A. G., Stevens K. R., Hafs H. D., I963. Characteristics of the hemolytic factor in bovine seminal plasma. J. Dairy Sci., 46, 618 (Abstract).

Libonati M., Floridi A., 1969. Breakdown of double-stranded RNA by bull semen ribonuclease. Eur. J. Biochem., 8, 8I-87.

Loor F., Forni L., Pernis B., I972. The dynamic state of the lymphocyte membrane factors affecting the distribution and turnover of surface immunoglobulins. Eur. J. Immunol., 2, 203-2I2.

Matousek J., 1964. Antigenic characteristics of spermatozoa from bulls, rams and boars. I. Erythrocyte antigens in bull spermatozoa. J. Reprod. Fertil., 8, I-3.

Matouse K J., r970. Hemolytic factor in bovine vesicle fluid. I. Individual differences in the sensitivity of cattle erythrocytes. Anim. Blood Groups. Biochem. Genet., 1, 89-93.

Matousex J., I972. Hemolytic factor in bovine vesicle fluid. 3. Inexistance of cattle erythrocyte sensitivity towards the hemolytic factor. Anim. Blood Groups. Biochem. Genet., 3, I5I-155.

Metz C., 1967. Gamete surface components and their role in fertilization. Fertilization ed. METZ C., Monroy A., Academic Press. New York, 1, 163-236.

Millar P. G., r956. Observations on the presence of a hemolysin in bovine semen. Brit. Vet. J., 112, I06-II5.

Multamaki S., Niemi M., I972. Trypsin like proteolytic activity in an a acrosomal extract of bull spermatozoa. Int. J. Fert., 17, 43-52.

Petrovsky E., I964. Hemolyticka aktivita semenné plasmy byku a a Kancu a jeji Vztah $K$ chladovému Soku spermii $Z$, ocisna vyroba, 9, 405-4I2. Cited by MatouseK J., I972.

Prakash C., I969. Unpublished.

Prakash C., I973a. Les alloantigènes sur les spermatozoïdes de mammifères. Leur contrôle par le génome haploïde. Ann. Génét. Sél. anim., 5, 507-5 I8.

PRAKASH C., I974 $b$. Séparation des spermatozoïdes mobiles des autres cellules et des débris cellulaires présents dans le sperme bovin. Ann. Biol. Anim. Bioch. Biophys., 14, 363-369.

Premkumar E., Bhargava P. M., I972. Transcription and translation in bovine Spermatozoa. Nature (New Biol.), 240, I39-143. 
Schmid D. O., Conneally P. M., Stone W. H., I964. Blood Group antigens on bull spermatozoa. J. Anim. Sci., 23, I I98-I I99 (Abs.).

Sheid B., Wilson S. M., I970 a. Inhibition of transfer RNA methylases by bull semen. I. Localisation, identification and properties of the inhibitor. Biochem. Biophys. Acta, 224, 382-390.

Sheid B., Wilson S. M., I97o $b$. $t$-RNA methylase inhibitor (s) in bull sperm. 54 th Ann. of the Federation of American Societies for experimental biology, Atlantic City, April 12-17. Fed. Proc., 29, 3883 (Abstract).

Sheid B., Wilson S. M., r97 I a. Necessity for restudying nucleic acid and protein synthesis in mammalian semen. Nature, 232, 628-629.

Sheid B., Wilson S. M., I97I $b$. Non-enzymatic hydrolysis of transfer RNA by bull semen. Life Sci., Part. II, 9r9-926.

Stone W. H., I972. Communication personnelle.

Shulman S., 1971. Antigenicity and autoimmunity in sexual reproduction : A review. Clin. Exp. Immunol., 9, 267-288.

Shulman S., 1972. Immunologic barriers to fertility. Obstet. Gynecol. Survey, 27, 553-606.

Voisin G., Delaunay A., Barber M., r95I. Sur les lésions testiculaires provoquées chez le cobaye par iso- et auto-sensibilisation. Ann. Inst. Pasteur, 81, I-I5.

Vorsin G. A., 1958. Recherches expérimentales et considérations sur la nature et la spécificité immunologique des autoantigènes. Immunopathologie, I er Symposium international, Bale-Seelisberg, I958, p. $133-144$.

Voisin G. A., Toullet F., Voisin J., r964. Étude sur l'hypersensibilité. 3. Caractère général du phénomène d'augmentation de perméabilité vasculaire au niveau des réactions d'hypersensibilité de type retardé. Ann. Inst. Pasteur, 106, 353-379.

Voisin G. A., Toullet F., 1967. Studies on spermatozoa autoantigens in the guinea-pig. Int. Symp. Immunology of Spermatozoa and Fertilization Varna, Bulgaria, Sept. 27-29, r967.

Voisin G. A., Toullet F., r968. Étude sur l'orchite aspermato-génétique autoimmune et les autoantigènes de spermatozoïdes chez le cobaye. Ann. Inst. Pasteur, 144, 727-755.

Vorsin G. A., 1973. Communication personnelle.

Vojtiskova M., Polackova M., Pokorna Z., I969. Histocompatibility antigens on mouse spermatozoa. Folia. Biol., 15, 322-332.

Vulchanov V. H., Papivanov R., I972. Immunological data suggesting post segregational gene action in human spermatozoa. Proc. Int. Symp. The Genetics of the Spermatozoon, BEATTY R. A., GlueckSOHN-Waelsch S. ed., Univ, Edinburgh, r77-190.

Zaneveld L. J. D., Polakoski K. L., Williams W. L., r972. Properties of a proteolytic enzyme from rabbit sperm acrosomes. Biol. Reprod., 6, 30-39. 